\title{
Inversion of Magnetoresistance in Organic Semiconductors
}

\author{
J. D. Bergeson, ${ }^{1}$ V. N. Prigodin, ${ }^{1}$ D. M. Lincoln, ${ }^{2}$ and A. J. Epstein ${ }^{1,2}$ \\ ${ }^{1}$ Department of Physics, The Ohio State University, Columbus, Ohio 43210-1117, USA \\ ${ }^{2}$ Department of Chemistry, The Ohio State University, Columbus, Ohio 43210-1173, USA
}

(Received 1 August 2007; published 13 February 2008)

\begin{abstract}
We report that organic semiconductors such as $\alpha$-sexithiophene ( $\alpha$-6T) have magnetoresistance (MR) with unexpected sign changes; depending on applied voltage, temperature, and layer thickness, the resistance may either increase or decrease upon application of a small magnetic field $(<100 \mathrm{mT})$. We propose that MR and the inversion of MR are due to the role of hyperfine interaction in a magnetic field, as illustrated by the recombination-limited regime.
\end{abstract}

DOI: 10.1103/PhysRevLett.100.067201

The manipulation of electrical transport in semiconductor systems through small, applied magnetic fields is an important facet of the field of spintronics [1-3]. A variety of magnetic field effects (MFEs) have been reported for organic semiconductors [4-18]. Frankevich and coworkers reported MFEs in the photoconductivity of single crystals and thin films of organic semiconductors such as anthracene where the photoconductance changed approximately $1-4 \%$ under small magnetic field $[19,20]$. The groups of Kalinowski [10] and Wohlgenannt [12,14] have reported magnetoresistance (MR) from 3 to $10 \%$ at low fields for several organic semiconductors. We have shown [16] that by doping with Pt or Ir complexes, the MR vanishes as spin coherence is lost due to increased spinorbit coupling, and we have proposed a model of magnetoresistance by the interconversion of singlets and triplets (MIST). The MIST mechanism operates via the hyperfine interaction and accounts for the behavior of these materials. Hu et al. later reported the influence of spin-orbit coupling in diminishing the MR in organic semiconductor devices $[17,18]$.

In this Letter, we present the first report of the inversion of MR in the organic semiconductor $\alpha-6 \mathrm{~T}$ and determine that it is controlled by voltage, temperature, and thickness of the semiconducting layer. Using results for $\alpha-6 \mathrm{~T}$, we show that the MR can be accounted for using the framework of the MIST mechanism [16]. In this model, hyperfine coupling interconverts triplets into singlets and vice versa, while an applied magnetic field lifts triplet degeneracy by Zeeman splitting, thereby changing the interconversion rate and leading to carrier recombination and electrical transport that are magnetic field dependent. MR is defined as the change in current density $J$ as a function of magnetic field $H$, i.e. MR $=$ $[J(0)-J(H)] / J(H)$. For certain values of voltage, temperature, and sample thickness, the electrical resistance will decrease with increasing magnetic field, i.e., negative MR. By appropriately changing the operating conditions, an inversion may be achieved where the resistance will, in contrast, increase with increasing magnetic field. Usually $[12,16]$, the MR response of organic semiconductors is
PACS numbers: 75.47. $-\mathrm{m}$, 72.80.Le, 73.43.Qt, 85.65.+h

negative, but some materials under particular circumstances can show positive MR. The oligomer $\alpha-6 \mathrm{~T}$, used widely in thin-film transistors [21] and light-emitting devices [22], has been selected for this detailed study because inversion of MR is more commonly manifested in this material as compared to other organic semiconductors for which we have recorded similar behavior. We show here that the MIST mechanism accounts for the observed positive and negative MR in $\alpha$-6T, and other organic semiconductors with low spin-orbit coupling and bipolar transport.

Samples for this study were grown on glass substrates coated with indium tin oxide (ITO). The substrates were rigorously cleaned prior to introduction into an inert atmosphere glove box $\left(\mathrm{O}_{2}\right.$ and $\mathrm{H}_{2} \mathrm{O}$ concentrations $\left.<1 \mathrm{ppm}\right)$ for deposition of all subsequent layers. A hole injection layer of poly(3,4-ethylenedioxythiophene):poly(styrenesulfonate) (PEDOT), acquired from H. C. Starck, was deposited by spin coating. Samples were then transferred into a vacuum chamber $\left(\sim 10^{-6}\right.$ Torr $)$ within the glove box for thermal deposition of the subsequent thin-film layers: $\alpha$-6T, supplied by Sigma-Aldrich; calcium (Ca), a low work function metal; and aluminum (Al) to prevent oxidation of the $\mathrm{Ca}$. The device architecture then consisted of stacked thin films of ITO/PEDOT $/ \alpha-6 \mathrm{~T} / \mathrm{Ca} / \mathrm{Al}$, where electrical contact was made to the ITO and Al. Magnetotransport measurements were conducted in a Quantum Design PPMS-9. A Keithley 2400 SourceMeter was used to apply a constant voltage to the devices, and the resulting current was recorded as a function of a parallel magnetic field to determine the MR, though no angle dependence is observed.

At room temperature, $\alpha-6 \mathrm{~T}$ exhibits both positive and negative MR, depending on the thickness of the semiconducting layer and on the applied voltage. For example, a device with a $50 \mathrm{~nm} \alpha$-6T layer [Fig. 1(a)] shows a negative MR at a low applied voltage of $2.0 \mathrm{~V}$, but as the voltage is increased slightly, the MR response becomes smaller until at $2.75 \mathrm{~V}$, it changes sign and becomes positive. The response continues to grow in the positive as the voltage increases to $3.0 \mathrm{~V}$. 

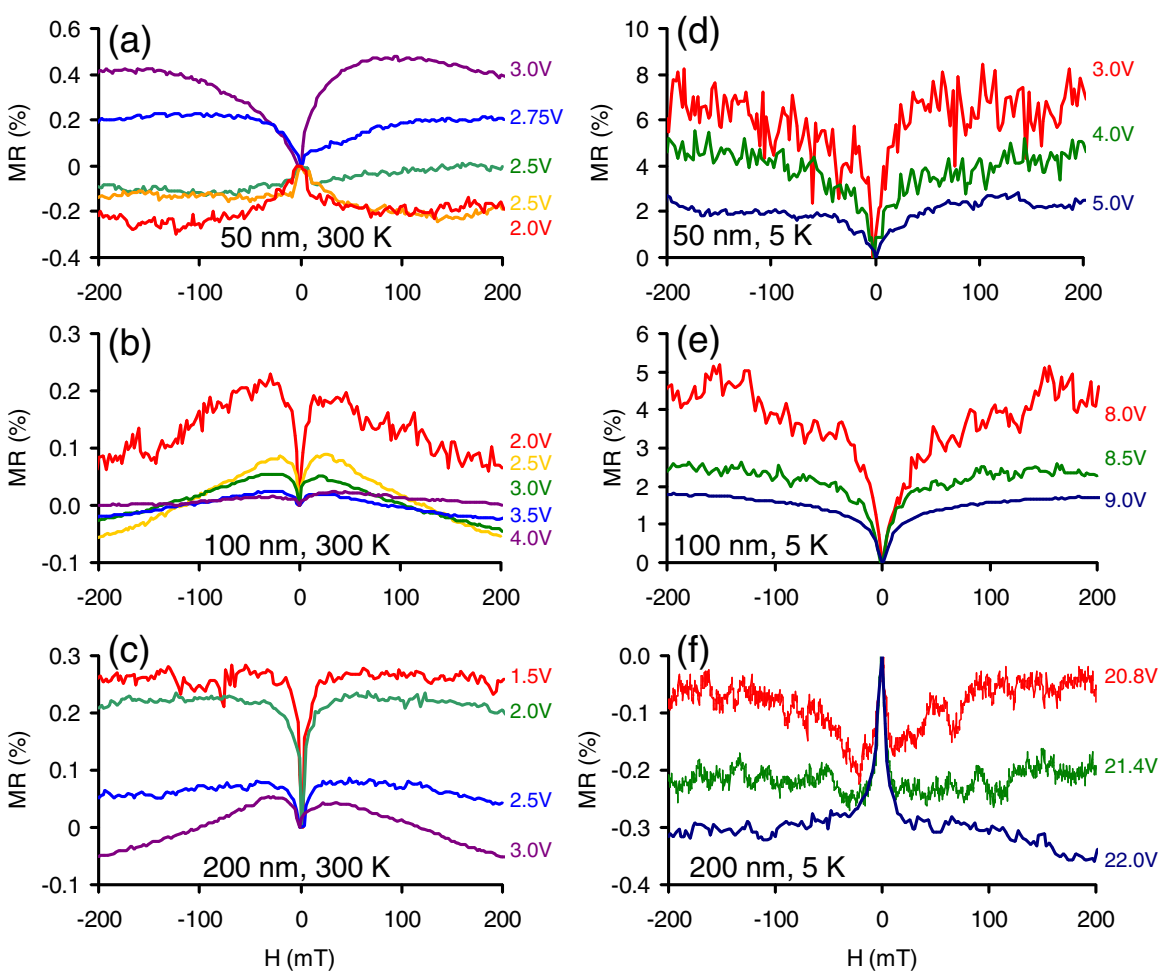

FIG. 1 (color online). MR for devices with $\alpha$-6T layers of varying thickness. Panels (a)-(c) show MR at $300 \mathrm{~K}$ for devices with $\alpha$-6T layers of (a) $50 \mathrm{~nm}$, (b) $100 \mathrm{~nm}$, and (c) $200 \mathrm{~nm}$, while panels (d)-(f) show MR at $5 \mathrm{~K}$ for layer thicknesses of (d) $50 \mathrm{~nm}$, (e) $100 \mathrm{~nm}$, and (f) $200 \mathrm{~nm}$. Shown in (a) and (c) are examples of MR inversion with applied bias, from negative to positive MR for (a) and vice versa for (c). The magnitude of the MR in (d) and (e) decreases as bias is increased. The MR in (f) is a mixture of positive and negative behavior that becomes strictly negative as bias increases.
At a thickness of $100 \mathrm{~nm}$ [Fig. 1(b)], room-temperature MR curves have a mixed positive and negative character. At low field $(<25 \mathrm{mT})$, each curve has positive character, but at higher fields, the MR is negative. In the range of 2.0$4.0 \mathrm{~V}$, the device produces a maximum positive $\mathrm{MR}$ at $2.0 \mathrm{~V}$. As voltage is increased, the magnitude of the lowfield positive MR decreases. However, the higher-field, negative MR also decreases in magnitude. In essence, the device is becoming less sensitive to magnetic field.

For an $\alpha-6 \mathrm{~T}$ layer thickness of $200 \mathrm{~nm}$ [Fig. 1(c)], still at $300 \mathrm{~K}$, the MR is positive at an initial, low applied voltage of $1.50 \mathrm{~V}$. At $2.0 \mathrm{~V}$, the MR is reduced slightly and more substantially at $2.5 \mathrm{~V}$. At $3.0 \mathrm{~V}$, the positive MR character is seen at low magnetic fields $(H<30 \mathrm{mT})$, but MR crosses through zero and becomes negative at higher values of $H$. This behavior seems to contrast that observed for the $50 \mathrm{~nm}$ layer.

When these 50, 100, and $200 \mathrm{~nm}$ devices are cooled to $5 \mathrm{~K}$, the semiconductor shows related behavior. For $\alpha-6 \mathrm{~T}$ layers of both 50 and $100 \mathrm{~nm}$ [Figs. 1(d) and 1(e)], a positive MR signal is observed, and the size of the response decreases with increasing voltage.

For the $200 \mathrm{~nm}$ device [Fig. 1(f)], again at $5 \mathrm{~K}$, the MR at low fields $(<10 \mathrm{mT})$ is negative. With $20.8 \mathrm{~V}$ applied across the device, a negative MR of $-0.15 \%$ is apparent at low fields and has a slight upturn as the field increases above $25 \mathrm{mT}$.

In order to analyze the magnetic-field dependence of these devices operating in regimes where electron-hole recombination contributes significantly to the current, it is mathematically convenient to define the quantity $\mu_{r}$, the recombination mobility, as $\mu_{r}=\epsilon \mathcal{B} /(2 e)$ where $\epsilon$ is the dielectric constant, $e$ is the elementary charge, and $\mathcal{B}$ is the total recombination rate coefficient. The parameter $\mu_{r}$ embodies the recombination kinetics and is helpful in deriving the current dependence, as described below. The recombination of charge carriers is a multistep process beginning with electrons and holes forming correlated pairs, described by the formation rate coefficient $b$ (the bimolecular recombination coefficient). For Coulombicallyinteracting, hopping electrons and holes, $b$ is given by the Langevin equation [23,24], $b=e\left(\mu_{e}+\mu_{h}\right) / \epsilon$. The formed electron-hole $(e-h)$ pair may then either recombine or dissociate [25], with pair recombination rate coefficient $k$ or dissociation rate coefficient $q$, respectively, leading to a total recombination rate coefficient given by $\mathcal{B}=$ $b k /(k+q)$. The fate of an $e-h$ pair is largely determined by the spin configuration with singlet recombination rates far exceeding those for triplets.

Within the MIST model, MR arises due to the sensitivity of carrier recombination to magnetic field. Uncorrelated electrons and holes form Coulombically-bound pairs with equal probability of being in a singlet $\left(S_{0}\right)$ or one of the three triplet configurations $\left(T_{1}, T_{0}, T_{-1}\right)$. Low spin-orbit coupling in $\alpha-6 \mathrm{~T}$ provides long spin relaxation times, while $e-h$ pairs, when spatially separated, have degenerate singlet and triplet states due to negligible exchange interaction. A key to this effect is sufficient hyperfine interaction to produce mixing between degenerate singlet and triplet states. At zero magnetic field, the singlet level is degenerate with the entire triplet manifold and mixing occurs among the four states. However, in the presence of a magnetic field, the degeneracy of the triplet states is lifted by Zeeman splitting, and interconversion only occurs 
between the $m=0$ states, while the long-lived triplets in the $m= \pm 1$ states no longer interconvert with the singlet and have a reasonable likelihood of dissociating. As a result, the application of a magnetic field can decrease $\mu_{r}$ by up to a factor of 2 .

For voltage $V$ applied across a semiconductor layer of thickness $d$ having electron and hole mobilities, $\mu_{e}$ and $\mu_{h}$, respectively, and operating in the space-charge-limited current (SCLC) regime, the current density $J$ is inversely proportional to the square root of $\mu_{r}$ [16]:

$$
J=\frac{3 \epsilon}{4}\left[\frac{2 \pi \mu_{e} \mu_{h}\left(\mu_{e}+\mu_{h}\right)}{\mu_{r}}\right]^{1 / 2}\left(\frac{V^{2}}{d^{3}}\right) .
$$

The SCLC regime is used as a point of departure for analyzing MR in $\alpha$-6T and other semiconductors; however, even in transport regimes where recombination is not the only limiting mechanism, e.g., injection-limited current [26], the MIST mechanism still applies. As we discussed, $\mu_{r}$ may be decreased by applying a magnetic field, which, in the SCLC regime of Eq. (1), leads to an increase in current. However, as $\mu_{r}$ is further decreased, the electron and hole carrier densities will continue to increase, but cannot do so indefinitely, and the current, as a function of $\mu_{r}$, will reach a maximum. Combinations of material parameters and operating conditions that produce relatively small values of $\mu_{r}$ result in current densities that are linearly dependent on $\mu_{r}$

$$
J=\mu_{r} \operatorname{dnp}\left(\frac{2 e^{2}}{\epsilon}\right) e^{\left(e V / k_{B} T\right)}
$$

and give positive MR. By varying the bias or other conditions, a device may operate under different transport regimes [27]. The current densities of Eq. (1) and (2) are connected through a crossover regime described by the critical recombination mobility $\mu_{c}$ and is determined by the MIST model as [28]

$$
\mu_{c}=\left[2 \pi \mu_{e} \mu_{h}\left(\mu_{e}+\mu_{h}\right)\right]^{1 / 3}\left[\frac{3 \epsilon^{2} V^{2}}{8 e^{2} n p d^{4}}\right]^{2 / 3} e^{\left(-2 e V / 3 k_{B} T\right)} .
$$

Here, $k_{B}$ is Boltzmann's constant, $T$ is the temperature, $d$ is the semiconductor layer thickness, and $n$ and $p$ are the electron and hole carrier densities. Figure 2(a) depicts the regimes described by Eqs. (1) and (2) with three example curves of $J$ as a function of $\mu_{r}$. The shaded region represents the range $\Delta \mu_{r}$ through which $\mu_{r}$ will vary as magnetic field is swept with all other parameters held constant. Figure 2(b) shows the essential behavioral characteristics of $\mu_{c}$ as a function of $V$ determined by Eq. (3). The dashed lines separate the three regions I, II, and III, each representing different magnetic field dependencies of $\mu_{r}$ producing various possible MR behaviors. The portion of the $J\left(\mu_{r}\right)$ curve that lies within $\Delta \mu_{r}$ is determined largely by the potential drop across the device, layer thickness, and temperature. Thus, the MIST model predicts that the current density shows the lowest sensitivity to magnetic field

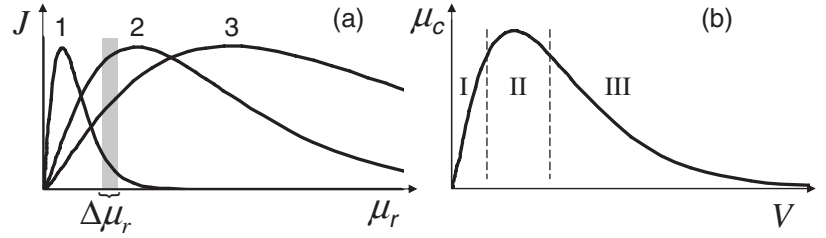

FIG. 2. (a) Schematic plot of curves corresponding to low (curve 1), intermediate (curve 2), and high (curve 3) values of $\mu_{c}$, where $\mu_{c}$ is the peak of each curve. The shaded region indicates the accessible range of $\mu_{r}\left(\Delta \mu_{r}\right)$. The portion of a given curve within the shaded region determines whether the MR will be negative, positive, or a mixture. (b) Plot of the critical recombination mobility $\mu_{c}$ as a function of applied voltage $V$ [Eq. (3)]. The different regions I, II, and III indicate where the function has a positive slope, a maximum, or a negative slope, respectively. The parameters voltage, temperature, and layer thickness determine the curve in which the device operates.

when the peak of the $J\left(\mu_{r}\right)$ curve is within $\Delta \mu_{r} . J\left(\mu_{r}\right)$ has the highest sensitivity when the inflection point of the curve is within $\Delta \mu_{r}$. For example, if for given values of $V, T$, and $d$ the recombination mobility is such that $\mu_{r}>$ $\mu_{c}$ for all magnitudes of magnetic field, then this situation corresponds to curve 1 of Fig. 2(a), giving negative MR. The portion of curve 1 within the shaded region, $\Delta \mu_{r}$, corresponds to space-charge-limited currents where $J$ is inversely proportional to the square root of $\mu_{r}$ [Eq. (1)].

As the voltage is increased, $\mu_{c}$ will increase in magnitude through region I until reaching a maximum in region II of Fig. 2(b), corresponding to an inversion from negative to positive MR, or to a shift from curve 1 to curve 3 in Fig. 2(a). In principle, at still higher voltages, another MR inversion could occur, from positive to negative as $\mu_{c}$ decreases in magnitude.

This framework is useful in understanding the change from negative to positive MR in the $50 \mathrm{~nm} \alpha$-6T layer [Fig. 1(a)]. At $300 \mathrm{~K}, \mu_{c}$ increases with increasing applied voltage, as expected for a sample in region I of Fig. 2(b). Under a $2.0 \mathrm{~V}$ bias, $\mu_{c}$ is relatively low, and the device is operating in the negative slope portion of curve 1 in Fig. 2, producing negative MR. As voltage is increased, $\mu_{c}$ shifts to the right, through curve 2 until at $3.0 \mathrm{~V}, \mu_{c}$ is greater than values of $\mu_{r}$ within $\Delta \mu_{r}$ and has shifted to the position of curve 3 , resulting in positive MR.

The explanation of the positive MR turning negative for the $200 \mathrm{~nm}$ layer at $300 \mathrm{~K}$ is similar to that for the MR in the $50 \mathrm{~nm}$, except that this sample is apparently operating in region III of Fig. 2(b). With $1.5 \mathrm{~V}$ applied, $\Delta \mu_{r}$ lies on curve 3 where $\partial J / \partial \mu_{r}$ is positive. The MR reduces as voltage is increased and the peak in Fig. 2(a) moves toward $\Delta \mu_{r}$. The mixed positive-negative $\mathrm{MR}$ at $3.0 \mathrm{~V}$ arises as the peak of the curve is within the region of $\Delta \mu_{r}$. The point on curve 2 corresponding to zero magnetic field at the right edge of $\Delta \mu_{r}$, but as the magnetic field is increased, the corresponding point on curve 2 moves to the left of the peak giving a temporary rise in current density followed by a drop. 
The mixed positive and negative behavior of the $100 \mathrm{~nm}$ $\alpha$-6T layer [Fig. 1(b)] is attributed to $\mu_{c}$ falling within the range of $\Delta \mu_{r}$, such that at low field, the MR is negative where $\partial J / \partial \mu_{r}$ is negative, but at higher fields the MR is positive where the slope of $J\left(\mu_{r}\right)$ is positive. This situation corresponds to that of curve 2 in Fig. 2(a).

At $5 \mathrm{~K}$, the MR behaviors of the 50 and $100 \mathrm{~nm}$ layers are very similar [Fig. 1(d) and 1(e)]; they are positive and decrease with increasing voltage in each sample which corresponds to a situation similar to curve 3 in Fig. 2(a). The decreasing MR with increasing voltage then originates from $\mu_{c}$ likewise decreasing, as in region III of Fig. 2(b), which results in the peak of curve 3 in Fig. 2(b) being closer to $\Delta \mu_{r}$ resulting in a lower sensitivity to magnetic field (i.e., $\left|\partial J / \partial \mu_{r}\right|$ becomes smaller).

The $200 \mathrm{~nm} \alpha$-6T layer at $5 \mathrm{~K}$ [Fig. 1(f)] generates an MR response that is roughly an order of magnitude smaller than that produced by the 50 and $100 \mathrm{~nm}$ layers. This is an indication that $\Delta \mu_{r}$ overlaps the peak of the $J\left(\mu_{r}\right)$ function, as in curve 2 . With $20.8 \mathrm{~V}$ applied, the point on curve 2 corresponding to zero magnetic field begins at the right of the peak producing small, negative MR, but as the magnetic field in increased $\mu_{r}$ moves over the peak to the left side and the character of the MR becomes positive. At the higher potentials of 21.4 and $22.0 \mathrm{~V}, \mu_{c}$ has decreased somewhat, shifting the peak to the left of $\Delta \mu_{r}$ resulting in strictly negative MR with increased magnitude owing to a higher value of $\left|\partial J / \partial \mu_{r}\right|$.

In addition to the oligomer $\alpha-6 \mathrm{~T}$, we have observed similar variations in MR amplitude and sign with temperature, thickness, and voltage in other organic, nonmagnetic semiconductors, such as the small molecule tris(8-hydroxyquinoline) aluminum $\left(\mathrm{Alq}_{3}\right)$, the poly(phenylene vinylene) derivative "Super Yellow," and $\operatorname{poly}(N$-vinylcarbazole).

We note that MR $<50 \%$ indicates that not all carriers participate in recombination and some carriers pass through the organic semiconductor to the counter electrode and therefore do not contribute to any magnetic field effects. Even in the presence of such nonideal conditions, the MIST mechanism applies to the recombination current.

In summary, we have performed an experimental study of the MR sign inversion from negative to positive, and vice versa, of organic semiconductors, with $\alpha-6 \mathrm{~T}$ as the model material, as a function of applied voltage, temperature, and film thickness and proposed the applicability of the MIST model in which the MR is governed by the nature of the dependence of the current density on the magneticfield-sensitive parameter $\mu_{r}$, the recombination mobility. The findings of this study apply widely to organic semiconductors owing to their long spin relaxation times, bipolar transport, and sufficiently strong hyperfine coupling to allow interconversion of singlet and triplet states.

We thank Aldrich Materials Science of Sigma-Aldrich Corp. for initial supply of $\alpha-6 \mathrm{~T}$, and Covion Organic Semiconductors GmbH, presently Merck OLED Materials GmbH, for supply of "Super Yellow." We ac- knowledge support from DOE Grant Nos. DE-FG0286ER45271 and DE-FG02-01ER45931 and AFOSR Grant No. FA9550-06-1-0175. We also acknowledge travel support from NSF Grant No. DMR-0645461.

[1] S. Wolf, D. Awschalom, R. Buhrman, J. Daughton, S. von Molnár, M. Roukes, A. Chtchelkanova, and D. Treger, Science 294, 1488 (2001).

[2] I. Žutić, J. Fabian, and S. Das Sarma, Rev. Mod. Phys. 76, 323 (2004).

[3] G. A. Prinz, Science 282, 1660 (1998).

[4] E. Frankevich and E. Balabanov, JETP Lett. 1, 169 (1965).

[5] R. Johnson, R. Merrifield, P. Avakian, and R. Flippen, Phys. Rev. Lett. 19, 285 (1967).

[6] R. Groff, R. Merrifield, A. Suna, and P. Avakian, Phys. Rev. Lett. 29, 429 (1972).

[7] C. E. Chidsey, L. Takiff, R. A. Goldstein, and S. G. Boxer, Proc. Natl. Acad. Sci. U.S.A. 82, 6850 (1985).

[8] V. N. Prigodin, N.P. Raju, K. I. Pokhodnya, J.S. Miller, and A. J. Epstein, Adv. Mater. 14, 1230 (2002).

[9] V. Dediu, M. Murgia, F. Matacotta, C. Taliani, and S. Barbanera, Solid State Commun. 122, 181 (2002).

[10] J. Kalinowski, M. Cocchi, D. Virgili, P. Di Marco, and V. Fattori, Chem. Phys. Lett. 380, 710 (2003).

[11] Z. Xiong, D. Wu, Z. V. Vardeny, and J. Shi, Nature (London) 427, 821 (2004).

[12] T. Francis, Ö. Mermer, G. Veeraraghavan, and M. Wohlgenannt, New J. Phys. 6, 185 (2004).

[13] A. H. Davis and K. Bussmann, J. Vac. Sci. Technol. A 22, 1885 (2004).

[14] Ö. Mermer, G. Veeraraghavan, T. L. Francis, Y. Sheng, D. T. Nguyen, M. Wohlgenannt, A. Köhler, M. K. Al-Suti, and M. S. Khan, Phys. Rev. B 72, 205202 (2005).

[15] M. Reufer, M. J. Walter, P. G. Lagoudakis, A. B. Hummel, J. S. Kolb, H. G. Roskos, U. Scherf, and J. M. Lupton, Nat. Mater. 4, 340 (2005).

[16] V. N. Prigodin, J. D. Bergeson, D. M. Lincoln, and A. J. Epstein, Synth. Met. 156, 757 (2006).

[17] Z. Xu, Y. Wu, and B. Hu, Appl. Phys. Lett. 89, 131116 (2006).

[18] Y. Wu and B. Hu, Appl. Phys. Lett. 89, 203510 (2006).

[19] E. Frankevich, Sov. Phys. JETP 23, 814 (1966).

[20] E. Frankevich, A. Zakhidov, K. Yoshino, Y. Maruyama, and K. Yakushi, Phys. Rev. B 53, 4498 (1996).

[21] A. Dodabalapur, H. Katz, L. Torsi, and R. Haddon, Science 269, 1560 (1995).

[22] G. Horowitz, P. Delannoy, H. Bouchriha, F. Deloffre, J.-L. Fave, F. Garnier, R. Hajlaoui, M. Heyman, F. Kouki, and P. Valat et al., Adv. Mater. 6, 752 (1994).

[23] U. Albrecht and H. Bässler, Phys. Status Solidi B 191, 455 (1995).

[24] L. J. A. Koster, V. D. Mihailetchi, and P. W. M. Blom, Appl. Phys. Lett. 88, 052104 (2006).

[25] C. L. Braun, J. Chem. Phys. 80, 4157 (1984).

[26] M. A. Baldo and S. R. Forrest, Phys. Rev. B 64, 085201 (2001).

[27] H. C. F. Martens, W. F. Pasveer, H. B. Brom, J. N. Huiberts, and P. W. M. Blom, Phys. Rev. B 63, 125328 (2001).

[28] V. N. Prigodin et al. (to be published). 